\title{
AUTOMOBILE AERODYNAMICS INFLUENCED BY AIRFOIL-SHAPED REAR WING
}

\author{
A. BULJAC, I. DŽIJAN, I. KORADE, S. KRIZMANIĆ and H. KOZMAR* \\ Faculty of Mechanical Engineering and Naval Architecture, University of Zagreb, Ivana Lučića 5, \\ 10000 Zagreb, Croatia
}

(Received 3 March 2015; Revised 4 September 2015; Accepted 29 November 2015)

\begin{abstract}
Computational model is developed to analyze aerodynamic loads and flow characteristics for an automobile, when the rear wing is placed above the trunk of the vehicle. The focus is on effects of the rear wing height that is investigated in four different positions. The relative wind incidence angle of the rear wing is equal in all configurations. Hence, the discrepancies in the results are only due to an influence of the rear wing position. Computations are performed by using the Reynolds-averaged Navier-Stokes equations along with the standard $k-\varepsilon$ turbulence model and standard wall functions assuming the steady viscous fluid flow. While the lift force is positive (upforce) for the automobile without the rear wing, negative lift force (downforce) is obtained for all configurations with the rear wing in place. At the same time, the rear wing increases the automobile drag that is not favorable with respect to the automobile fuel consumption. However, this drawback is not that significant, as the rear wing considerably benefits the automobile traction and stability. An optimal automobile downforce-to-drag ratio is obtained for the rear wing placed at $39 \%$ of the height between the upper surface of the automobile trunk and the automobile roof. Two characteristic large vortices develop in the automobile wake in configuration without the rear wing. They vanish with the rear wing placed close to the trunk, while they gradually restore with an increase in the wing mounting height.
\end{abstract}

KEY WORDS : Automobile aerodynamics, Rear wing, Aerodynamic forces, Steady Reynolds-averaged Navier-Stokes equations, $k-\varepsilon$ turbulence model

\section{INTRODUCTION}

Various technical solutions have been explored to optimize fuel consumption of road vehicles as well as to improve their traction and stability. An increase in the maximum tire lateral force, which is improving car traction and cornering ability, can be achieved by increasing the tire normal force (Katz, 1996). This can be obtained by redesigning the automobile shape using various aerodynamic devices. These devices generally neutralize the uplift force and produce the downforce, thus increasing the tire normal force and improving the vehicle dynamics without increasing the actual mass of the car. One of the important aerodynamic parts commonly used to improve automobile aerodynamics is an airfoil-shaped rear wing placed at the trunk of the vehicle. While the basic aerodynamic characteristics of the rear wing are generally known, further work is still required with respect to the wind incidence angle and the wing mounting height at the trunk of the automobile. The general goal is to increase the automobile downforce to improve its traction and stability, while simultaneously try to avoid considerable drag increment, which adversely increases fuel consumption.

*Corresponding author. e-mail: hrvoje.kozmar@fsb.hr
While the automobile aerodynamics has been traditionally studied experimentally in the wind tunnels, the Computational Fluid Dynamics (CFD) has been increasingly used to investigate this topic, even though further improvements are still necessary to make this approach more reliable (Guilmineau, 2008). Chien-Hsiung et al. (2009) studied influence of a rear wing on aerodynamic behavior of a passenger car. Their CFD results indicate an improved vertical stability of the vehicle in configurations with a rear wing. The two dominant rear vortices (C-pillar vortices) in the wake of the automobile, developing from the vertical supports of an automobile window area and adversely influencing an overall vehicle aerodynamics, reduce significantly in size when the rear wing is used (Bao, 2011), while the wing shape proved to be an important issue as well (Norwazan et al., 2012).

While the rear wing has been commonly used to improve vehicle aerodynamics, various possibilities with the front wing and rear diffuser have been explored as well. $\mathrm{Hu}$ et al. (2011) reported a decrease in the lift coefficient with an increase in the rear diffuser angle with respect to ground. The use of a rear diffuser for the purposes of aerodynamic drag reduction of a sedan car was studied in CFD simulations by Kang et al. (2012), indicating a drag reduction of maximum $4 \%$ yielding a lower automobile 
fuel consumption. For the front wing, the wind incidence angle considerably influences flow characteristics around the vehicle (Diasinos and Gatto, 2008). In addition to sedan-type vehicles, the rear wings proved to be effective for trucks as well (Ha et al., 2011), while aerodynamically shaping the rear part of the vehicles can yield improvements in vehicle aerodynamics, even though the wings and spoilers are not used (Song et al., 2012). In addition to other important issues, wheel rotation is observed to influence aerodynamics of vehicles as well (Fackell and Harvey, 1975). For example, Rizal et al. (2012) report that tires account for more than $25 \%$ of the total drag coefficient for open-wheel cars.

In the past, vehicle aerodynamics has been commonly studied for quasi-steady flow conditions. However, in case of transient flow conditions the vehicle aerodynamics can change dramatically, which is particularly exhibited when vehicles are passing viaduct and bridges (Kozmar et al., 2012, 2015). The quasi-steady approach proved not to be completely adequate during an overtaking maneuver (Corin et al., 2008), as in this case the aerodynamic forces can be $400 \%$ larger than calculated using the quasi-steady analysis, which can have significant effect on vehicle stability.

The scope of this study is to computationally investigate influence of the rear-wing mounting height on aerodynamic properties of the sedan-type automobile and the flow characteristics in the wake of the automobile. The effects of the wind incidence angle on a studied airfoilshape wing are analyzed and an optimal wind incidence angle is determined with respect to downforce-to-drag ratio. The rear wing is placed on the car with four different heights in such way that relative wing incidence angle is equal for all setups, therefore the effects of wing angle of attack are negligible. Contributions of the automobile and the wing to the overall aerodynamic forces are analyzed for different setups.

\section{COMPUTATIONAL SETUP}

The studied automobile is BMW E38. It is simplified by introducing the vertical symmetry plane, i.e. the flow and aerodynamic forces are simulated on one (left-hand) side of the vehicle only, and the results are assumed to be symmetrical on the right-hand side of the vehicle. Nevertheless, as the preliminary computational tests indicate the symmetry of the flow with respect to automobile symmetry plane, while the automobile is symmetrical on both sides as well, this proved not to make any difference in the results. Small details of the automobile geometry, such as bumpers and mirrors, are not modeled, as they are not considered to influence the results notably, while modeling those details significantly increases complexity of the computational domain and therefore the time necessary to perform the computations.

As the focus of this study is on effects of the rear wing

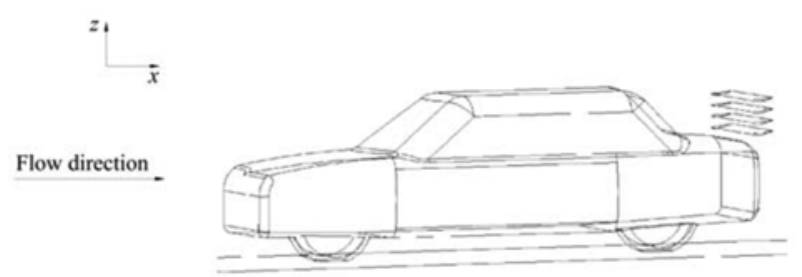

Figure 1. Geometrical model of the BMW E38 automobile model along with four different mounting heights of the rear wing.

mounting height on the flow characteristics and aerodynamic forces, in total five different computational models are created, i.e. one model without the rear wing and four models for various wing mounting heights. A schematic view of the studied automobile together with different positions of the airfoil-shaped rear wing is reported in Figure 1. In each simulation, only one rear wing is considered at the time.

Distance from the trunk to the roof of the automobile is constant and denoted as $H$, while the distance from the trunk to the wing leading edge, different for every wing mounting height, is $b$. These dimensions are presented in Figure 2. Dimensions of the computational domain are selected to keep the blockage of the computational domain below the commonly accepted critical value of $6 \%$ (West and Apelt, 1982). In particular, given the selected size of

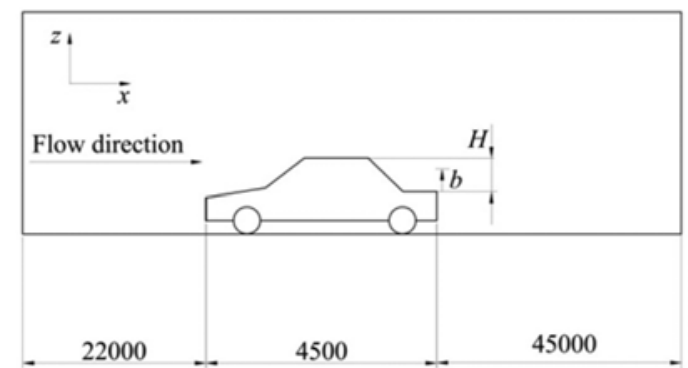

Figure 2. Schematic side view of the computational domain and the BMW E38 automobile model, dimensions are given in millimeters.

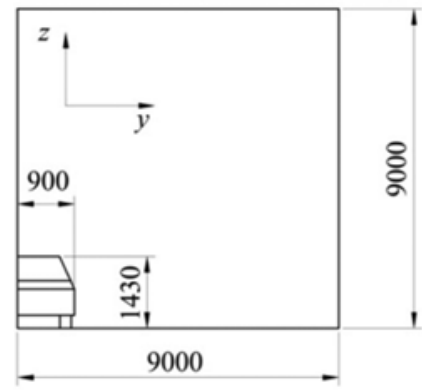

Figure 3. Schematic rear view of the computational domain and the BMW E38 automobile model, dimensions are given in millimeters. 


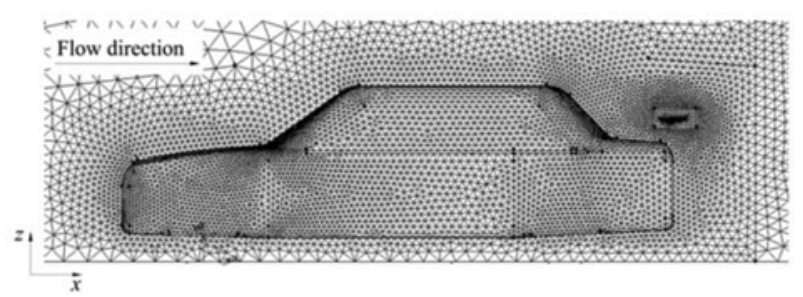

Figure 4. Geometrical discretization of the computational domain on smaller scale, automobile symmetry plane.

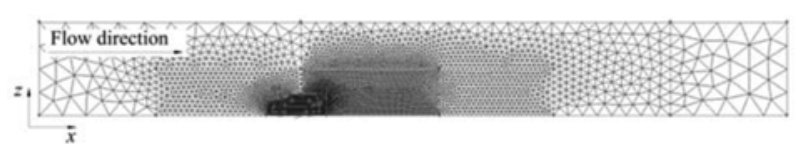

Figure 5. Geometrical discretization of the computational domain on larger scale, automobile symmetry plane.

the computational domain and the automobile reported in Figures 2 and 3, the automobile blockage is $1.3 \%$ of the computational domain which is considerably lower than $6 \%$.

Geometrical discretization of the computational domain is carried out with several blocks of unstructured tetrahedral volumes, while in the area of the rear wing unstructured hexahedral volumes are used. The mesh is finer in the areas where strong gradients of observed physical quantities are expected, Figures 4 and 5. The height of the first volume above the road and the car body is determined based on $y^{+}$values between 30 and 300 .

Five different meshes are created, i.e. one for each studied computational model. A number of control volumes for tested configurations is in the range from 404932 to 432509 control volumes. Rotation of front and rear wheels is simulated according to Fackell and Harvey (1975), while the road is defined as a moving wall with noslip condition. Hence, boundary layer is not developed on the ground, but only on the car body. This setup corresponds to the setup occurring on the road, as importance of these conditions is emphasized by Elofsson and Bannister (2002).

Computational model is developed in Fluent v15.0 commercial software for a stationary viscous fluid flow, by iterative solving of the RANS equations, along with the standard $k-\varepsilon$ turbulence model and the standard wall functions. As the focus of this study is on time averaged aerodynamic forces, transient aerodynamic effects are not analyzed here, while this remains to be addressed in some future study. Unsteady RANS equations for incompressible turbulent flow are given as;

a) Continuity equation,

$$
\frac{\partial \bar{v}_{\mathrm{j}}}{\partial x_{\mathrm{j}}}=0
$$

b) Momentum equation, $\frac{\partial \rho \bar{v}_{\mathrm{i}}}{\partial t}+\frac{\partial}{\partial x_{\mathrm{j}}}\left(\rho \bar{v}_{\mathrm{j}} \bar{v}_{\mathrm{i}}\right)=-\frac{\partial \bar{p}}{\partial x_{\mathrm{i}}}+\frac{\partial}{\partial x_{\mathrm{j}}}\left[\mu\left(\frac{\partial \bar{v}_{\mathrm{i}}}{\partial x_{\mathrm{j}}}+\frac{\partial v_{\mathrm{j}}}{\partial x_{\mathrm{i}}}\right)+\mu_{\mathrm{t}}\left(\frac{\partial \bar{v}_{\mathrm{i}}}{\partial x_{\mathrm{j}}}+\frac{\partial \bar{v}_{\mathrm{j}}}{\partial x_{\mathrm{i}}}\right)-\frac{2}{3} \rho \bar{k} \delta_{\mathrm{ij}}\right]$.

Components of the averaged velocity are denoted as $v_{\mathrm{i}}$, $\bar{p}$ is averaged pressure, $\rho$ is density, $x_{\mathrm{i}}$ are components of the position vector, $\mu$ is dynamic viscosity, $\mu_{\mathrm{t}}$ is turbulence viscosity, $\bar{k}$ is averaged turbulence kinetic energy

$\bar{k}=\frac{\overline{v_{\mathrm{i}}{ }^{\prime} v_{\mathrm{i}}{ }^{\prime}}}{2}$, while the turbulence viscosity is defined as;

$\mu_{\mathrm{t}}=C_{\mu} \rho \frac{\bar{k}^{2}}{\bar{\varepsilon}}$,

where $\bar{\varepsilon}$ is averaged dissipation of turbulence kinetic energy. This numerical model is used together with two additional transport equations of the $k-\varepsilon$ turbulence model (Launder and Spalding, 1974),

$$
\begin{aligned}
& \frac{\partial}{\partial t}(\rho \bar{k})+\frac{\partial}{\partial x_{\mathrm{j}}}\left(\rho \overline{v_{\mathrm{j}}} \bar{k}\right)=\frac{\partial}{\partial x_{\mathrm{j}}}\left[\left(\mu+\frac{\mu_{\mathrm{t}}}{\sigma^{\mathrm{k}}}\right) \frac{\partial \bar{k}}{\partial x_{\mathrm{j}}}\right]+G-\rho \bar{\varepsilon} \\
& \frac{\partial}{\partial t}(\rho \bar{\varepsilon})+\frac{\partial}{\partial x_{\mathrm{j}}}\left(\rho \overline{v_{\mathrm{j}}} \bar{\varepsilon}\right)=\frac{\partial}{\partial x_{\mathrm{j}}}\left[\left(\mu+\frac{\mu_{\mathrm{t}}}{\sigma^{\varepsilon}}\right) \frac{\partial \bar{\varepsilon}}{\partial x_{\mathrm{j}}}\right]-C_{1} G \frac{\bar{\varepsilon}}{\bar{k}}-C_{2} \rho \frac{\bar{\varepsilon}}{\bar{k}} .
\end{aligned}
$$

$G$ is generation of turbulence kinetic energy,

$$
G=\mu_{\mathrm{t}}\left(\frac{\partial \bar{v}_{\mathrm{i}}}{\partial x_{\mathrm{j}}}+\frac{\partial \bar{v}_{\mathrm{j}}}{\partial x_{\mathrm{i}}}\right) \frac{\partial \bar{v}_{\mathrm{i}}}{\partial x_{\mathrm{j}}}=\frac{1}{2} \mu_{\mathrm{t}}\left(\frac{\partial \bar{v}_{\mathrm{i}}}{\partial x_{\mathrm{j}}}+\frac{\partial \bar{v}_{\mathrm{j}}}{\partial x_{\mathrm{i}}}\right)\left(\frac{\partial \bar{v}_{\mathrm{i}}}{\partial x_{\mathrm{j}}}+\frac{\partial \bar{v}_{\mathrm{j}}}{\partial x_{\mathrm{i}}}\right) .
$$

Coefficients given in Equations (4) and (5) are constant for this turbulence model in case of large Reynolds numbers, while their standard values, also used in this work, are,

$C_{\mu}=0.09, \sigma^{\mathrm{k}}=1, \sigma^{\varepsilon}=1.3, C_{1}=1.44, C_{2}=1.92$.

Initial values of the turbulence kinetic energy and the dissipation of turbulence kinetic energy are selected to simulate a low turbulent flow, i.e. turbulence intensity is $0.1 \%$ and turbulence integral length scale is $0.01 \mathrm{~m}$,

$$
\bar{k}=0.0006 \mathrm{~m}^{2} / \mathrm{s}^{2}, \bar{\varepsilon}=0.0002415 \mathrm{~m}^{2} / \mathrm{s}^{3} .
$$

Freestream undisturbed flow velocity in all simulations is $20 \mathrm{~m} / \mathrm{s}$, the velocity profile is uniform, thus the Reynolds numbers and flow conditions are similar to the usual driving conditions. Preliminary tests carried out in an empty computational domain (without the automobile) indicate there are no flow modifications between the inlet to the computational domain and the automobile position. Calculations are performed using the first and second order upwind numerical schemes, with more details on these schemes provided in Ferziger and Perić (2002), while the criterion for interruption of the iterative process was that residuals of all equations being solved were smaller than $10^{-4}$.

In order to determine an optimal angle of the rear wing with respect to the flow, preliminary $2 \mathrm{D}$ simulations are performed to analyze flow characteristics around the 


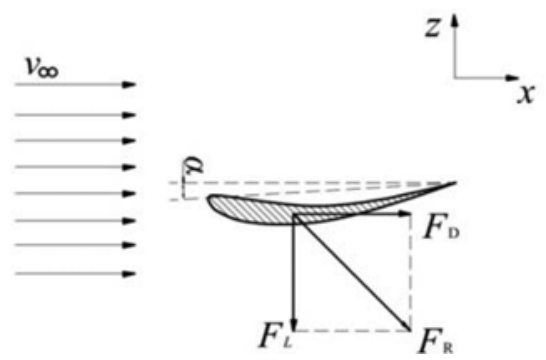

Figure 6. Computational setup for analyzing an optimal angle of aerofoil with respect to the flow.

selected airfoil shape, as lift and drag coefficients of the aerofoil are primarily influenced by the wind incidence angle, Figure 6. In this study, the term 'airfoil' is used for 2D aerodynamic profiles, while term 'aerofoil' is used for $3 \mathrm{D}$ aerodynamic profiles, as it is common in the standard aerodynamics textbooks.

Undisturbed freestream wind velocity is denoted as $v_{\infty}, \alpha$ is the wind incidence angle, $F_{\mathrm{D}}$ and $F_{\mathrm{L}}$ are drag and lift force, respectively, and $F_{\mathrm{R}}$ is the resultant force. Drag and lift forces are analyzed in a form of dimensionless coefficients,

$$
\begin{aligned}
& C_{\mathrm{D}}=\frac{F_{\mathrm{D}}}{\frac{1}{2} \rho \cdot v_{\infty}^{2} \cdot A_{\mathrm{D}}} \\
& C_{\mathrm{L}}=\frac{F_{\mathrm{L}}}{\frac{1}{2} \cdot \rho \cdot v_{\infty}^{2} \cdot A_{\mathrm{L}}},
\end{aligned}
$$

where $C_{\mathrm{D}}$ is drag coefficient, $C_{\mathrm{L}}$ is lift coefficient, $A_{\mathrm{D}}$ and $A_{\mathrm{L}}$ are reference areas for drag and lift coefficients, respectively. The coordinate system provided in Figure 6 is used for all the results reported in this study. Reference area used for calculating aerodynamic coefficients for aerofoil is cross-sectional area of the aerofoil as observed from above,

$$
A_{\mathrm{D}}=A_{\mathrm{L}}=0.27 \mathrm{~m}^{2} \text {. }
$$

Chord of the aerofoil is $150 \mathrm{~mm}$, while the span of the aerofoil is $1,800 \mathrm{~mm}$. When the overall aerodynamic force coefficients are considered for the automobile, it needs to be mentioned that the reference frontal area used to calculate aerodynamic coefficients is the vertical plane projection of the left-hand side of the automobile only,

$A_{\mathrm{D}}=A_{\mathrm{L}}=1.05 \mathrm{~m}^{2}$.

\section{RESULTS AND DISCUSSION}

The 2D computational simulations are performed to investigate the effects of the wind incidence angle on aerodynamic forces experienced by the selected aerofoil shape, when the aerofoil is placed in the undisturbed flow (not on the trunk of the automobile). The obtained lift and drag coefficients are reported in Figures 7 and 8.

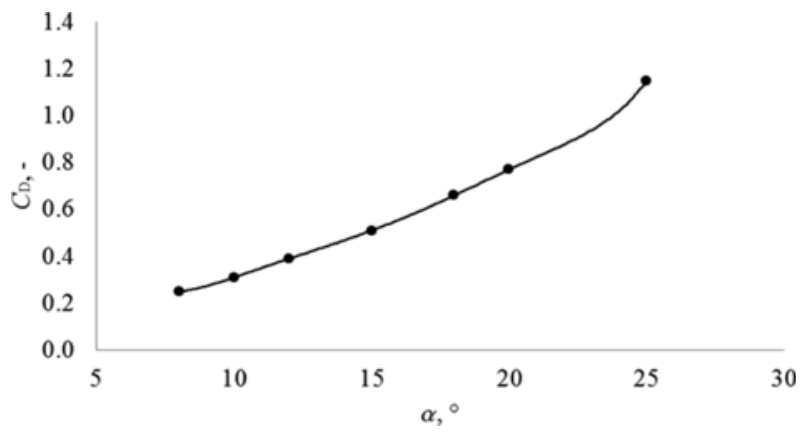

Figure 7. Aerofoil drag force coefficient as a function of the wind incidence angle; dots are CFD results, solid line is the best fit approximation.

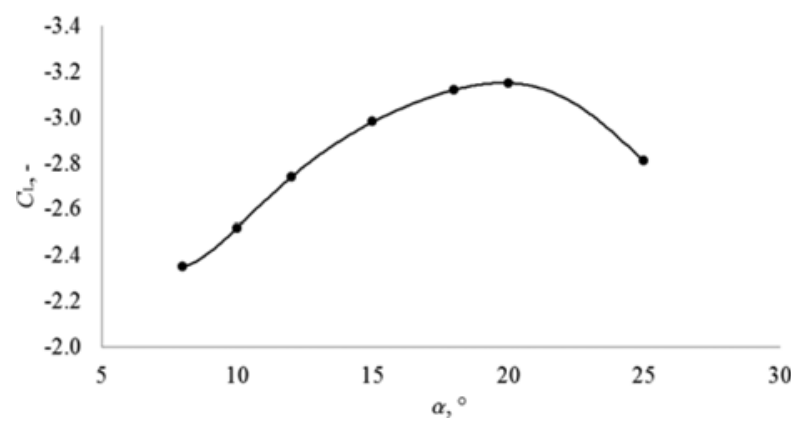

Figure 8. Aerofoil lift force coefficient as a function of the wind incidence angle; dots are CFD results, solid line is the best fit approximation.

It can be observed that the drag coefficient increases with an increase in the wind incidence angle, while the lift coefficient reaches its maximum at around $20^{\circ}$ and decreases with further increasing incidence angle, in accordance with McBeath (2011). In particular, with increasing the wind incidence angle, the frontal area of the aerofoil exhibiting strongly positive pressures becomes larger, while the lower leeward surface of the aerofoil characterized with negative pressures becomes larger as well due to flow separation closer to the leading edge. These both trends in turn contribute to increasing the drag, while simultaneously influencing the lift coefficient to behave as reported in Figure 8 .

Based on these results, $18^{\circ}$ is selected as the optimal wind incidence angle with respect to the aerofoil. In particular, while the maximum lift coefficient is obtained for $20^{\circ}$, it remains nearly the same for $18^{\circ}$. At the same time, the drag coefficient is considerably smaller for $18^{\circ}$ than it is the case for $20^{\circ}$. Hence, while the goal of this study is to optimize the maximum downforce with the minimum drag, $18^{\circ}$ is selected as the optimal aerofoil wind incidence angle for further analysis.

As the optimal wind incidence angle is selected for an aerofoil placed in the undisturbed, freestream flow, flow characteristics need to be determined next for the 

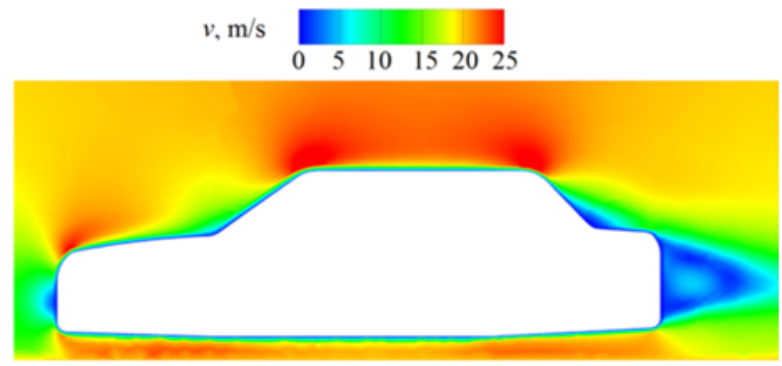

Figure 9. Mean velocity field in the automobile symmetry plane.

automobile without the rear wing in place in order to determine an optimal placing position of the rear wing on the trunk of the automobile. The respective mean velocity field in the area around the automobile is reported in the lateral symmetry plane in Figure 9, whereas the undisturbed free stream velocity is $20 \mathrm{~m} / \mathrm{s}$. The velocity field focusing on the trunk area only is reported in Figure 10 .

Above the trunk, there is a considerable velocity reduction, which diminishes with increasing the height. As the goal with placing the rear wing above the trunk is to increase the downforce, while not considerably increasing the drag, which is expected to be possible to be realized in the height range between the trunk surface and the roof height, four different height positions are selected and applied in further analysis. These positions are in the $b / H$ range from 0.17 to 0.83 , as the positions in this range are expected to yield a significant downforce, while the rear wing is not numerically tested in positions above the roof, as this is anticipated to adversely increase the drag of the automobile, whereas it is not optimal from the design point of view.

Therefore, the streamlines in the automobile symmetry plane in the positions where the rear wing is to be placed in

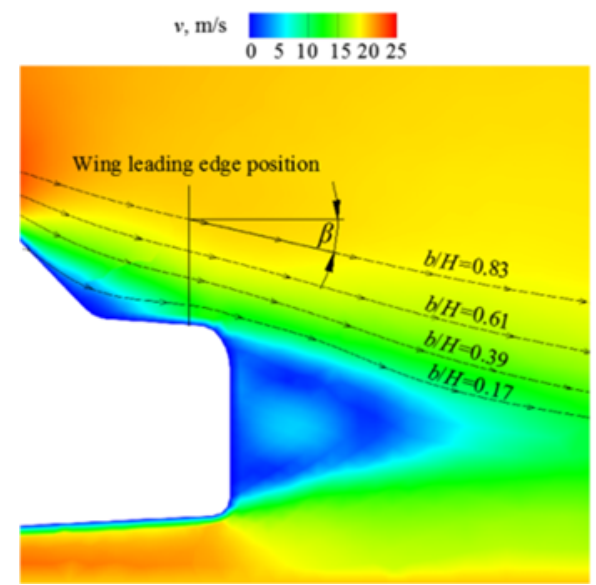

Figure 10. Flow streamlines in the automobile symmetry plane above the automobile trunk for selected rear wing positions.
Table 1. Flow angles $\beta$ between the streamlines and the horizontal axis.

\begin{tabular}{ccccc}
\hline$b / H,-$ & 0.17 & 0.39 & 0.61 & 0.83 \\
\hline$\beta,^{\circ}$ & $12.2^{\circ}$ & $13.4^{\circ}$ & $14.4^{\circ}$ & $15.2^{\circ}$ \\
\hline
\end{tabular}

further computational tests are presented in Figure 10, where the vertical line denotes the positions of the wing leading edge.

It can be observed that the flow incidence angle on a rear wing, which is to be placed on the automobile trunk in later simulations, will change when increasing the wing distance from the trunk, as the angles $\beta$ between the streamlines and the horizontal axis are reported in Table 1. While the wind incidence angle $18^{\circ}$ is adopted to be optimal with respect to aerodynamic forces experienced by the used airfoil, the rear wing will be placed at the trunk in a way that the wind incidence angle (taking into account the angles of the streamlines observed in Figure 10) is $18^{\circ}$.

Once the numerical setup with the rear wing placed above the automobile trunk is determined, simulations are performed in four different configurations, i.e. for the rear wing placed at four different heights above the trunk. The obtained lift force coefficients dependent on the height of the rear wing from the automobile trunk are presented in Figure 11.

Preliminary computational tests for the automobile without the rear wing indicate the lift force coefficient to be positive and equal to 0.07 , which indicates an existence of an upforce that is adverse for the automobile traction and stability. When the rear wing is mounted, the lift force coefficient becomes negative indicating an existence of a downforce, which generally benefits the automobile dynamic behavior. In particular, when increasing the rear wing height, the lift force coefficient increases up to $b / H=$ 0.61 and slightly decreases thereafter. This trend is generally comparable to Norwazan et al. (2012), ChienHsiung et al. (2009), as they reported lift reduction with integration of the rear wing as well. However, in Norwazan et al. (2012), Chien-Hsiung et al. (2009) the lift force is

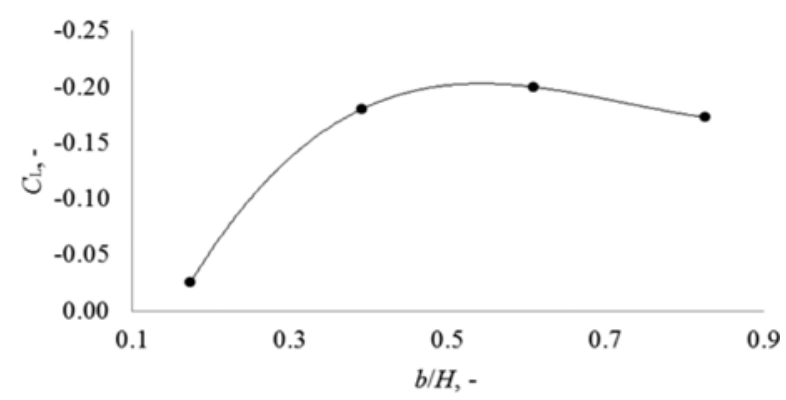

Figure 11. Lift force coefficient dependent on the height of the rear wing from the automobile trunk; dots are CFD results, solid line is the best fit approximation. 


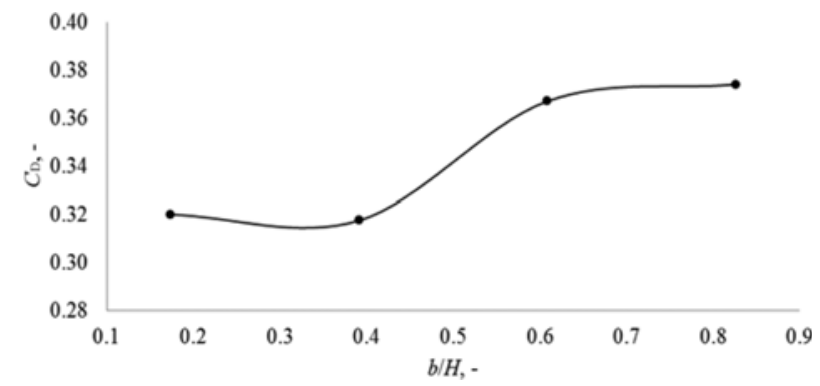

Figure 12. Drag force coefficient dependent on the height of the rear wing from the automobile trunk; dots are CFD results, solid line is the best fit approximation.

directed upwards in all configurations, while in the present study a downforce that enhances automobile dynamics even more is obtained, as a result of an optimization of the flow incidence angle onto the rear wing.

The drag force coefficient is equal to 0.29 for the automobile without the rear wing. When the rear wing is in place, the drag force coefficient is increased and nearly constant in range up to $b / H=0.39$. It increases with further increasing the rear wing height, thus indicating that placing the rear wing generally increases the drag of the automobile, Figure 12.

This increase in the drag force coefficient with increasing height is observed not to be linear. The drag force coefficient of the studied automobile without the rear wing is in good agreement with the BMW E38 factory specifications, where the drag force coefficient is reported to be 0.3 (Carfolio, 2015). Norwazan et al. (2012), ChienHsiung et al. (2009) reported as well that using the rear wing can increase the automobile drag coefficient.

The trends observed for the lift and drag force coefficients reported in Figures 11 and 12 are further analyzed by using the results for the flow field around the rear part of the automobile, Figure 13. It is particularly important to investigate the flow velocity close to the trunk surface, as it directly influences the pressure at the vehicle surface, which in turn characterizes the automobile drag and lift forces. The results are reported for the configuration without the rear wing $(b / H=0)$, as well as for the rear wing in place $(b / H=0.17 ; 0.39 ; 0.61 ; 0.83)$.

For the automobile without the rear wing, there is a region of low velocities observed at the rear windshield, above the trunk and downwind from the automobile (within the height range of the trunk). For the lowest rear wing position $(b / H=0.17)$, the flow velocity increases in the area immediately adjacent to the trunk surface that is particularly exhibited along the vertical trunk surface. The flow velocity close to the trunk surface becomes again smaller when the rear wing is placed higher from the trunk. Simultaneously, there is a localized region of very large velocities at the leading edge of the rear wing from the low side.

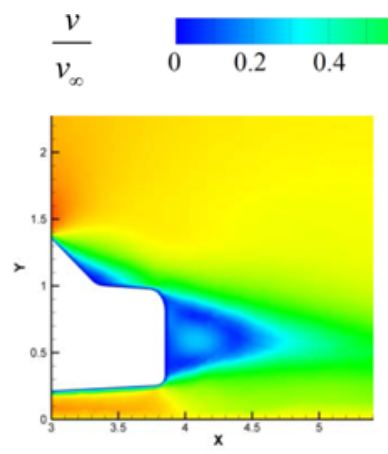

(a) $b / H=0$

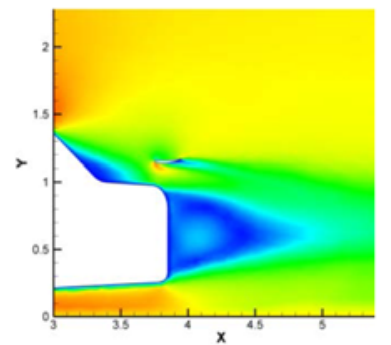

(c) $b / H=0.39$

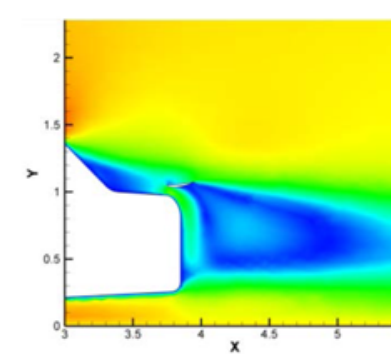

(b) $b / H=0.17$

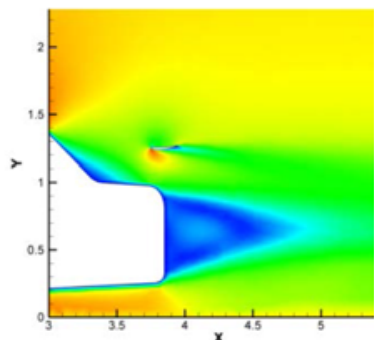

(d) $b / H=0.61$

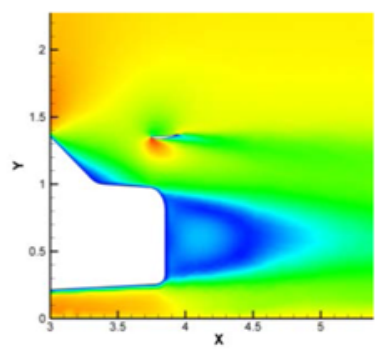

(e) $b / H=0.83$

Figure 13. Velocity field around the rear part of the automobile.

In order to distinguish contributions of the rear wing and the automobile to the overall drag force coefficient reported in Figure 12, the drag force coefficient is analyzed separately for the rear wing and the automobile in all numerically studied configurations with the rear wing in

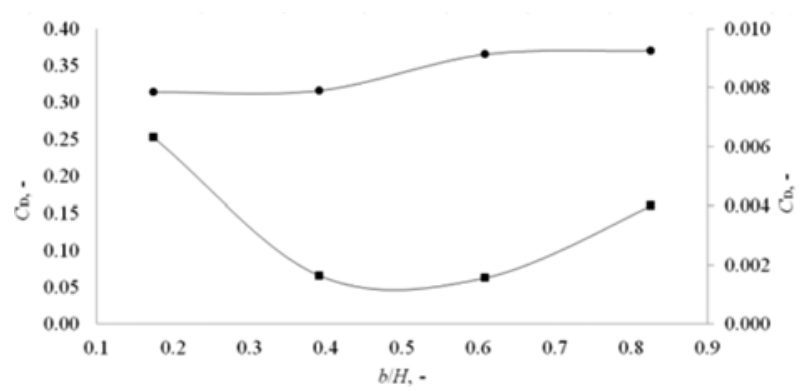

Figure 14. Contribution of the automobile and the rear wing to the overall drag force coefficient; dots are CFD results for the automobile body (left $y$-axis), squares are CFD results for the rear wing (right $y$-axis), solid lines are the best fit approximations. 
place, Figure 14. Both drag coefficients are calculated using the same reference area, as it is used for calculation of overall force coefficients.

The results indicate that the contribution of the automobile body to the overall drag force coefficient is dominant with respect to the wing contribution. The contribution of the automobile body to the overall drag is smaller and nearly constant for the wing mounting heights $b / H=0.17$ and 0.39 , while it is increased for two higher rear wing positions, i.e. for $b / H=0.61$ and 0.83 . Those results combined yield an optimal rear wing height of $b / H$ $=0.39$ with respect to the drag force.

The pressure coefficient distribution at the rear wing surface for different mounting heights is reported in Figure
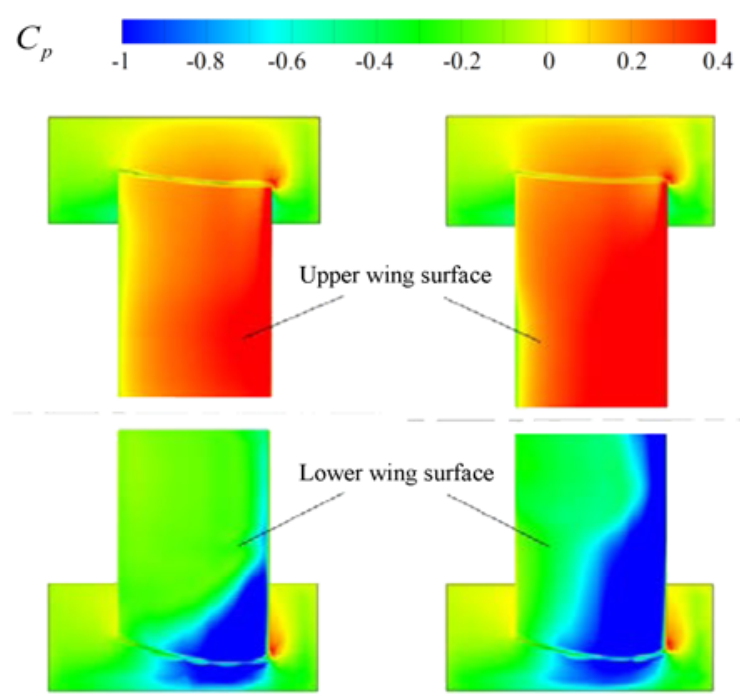

(a) $b / H=0.17$

(b) $b / H=0.39$
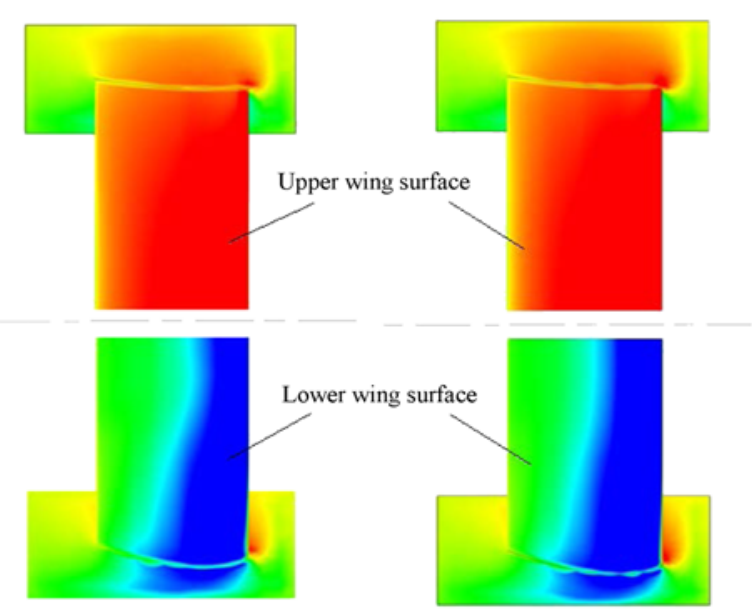

(c) $b / H=0.61$

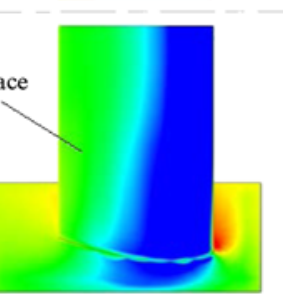

(d) $b / H=0.83$

Figure 15. Pressure coefficients at the rear wing surface for different wing mounting heights. Pressure coefficients on the upper wing surface are shown on the upper part of the each figure, while for the lower wing surface on the lower part of the figure.
15. The results in the first row of Figures 15 (a) (d) are reported for the upper side of the rear wing (the rear wing surface observed from above), while the results in the second row are presented for the lower side of the rear wing (the rear wing surface observed from below). The Figure 15 serves to analyze the trends in the lift force coefficient of the rear wing alone, when increasing the height of the rear wing with respect to the trunk surface. The dimensionless pressure coefficient is defined as;

$C_{\mathrm{p}}=\frac{p-p_{\infty}}{\frac{1}{2} \rho v_{\infty}^{2}}$

where $p$ is pressure at the rear wing surface, $p_{\infty}$ is pressure of the undisturbed freestream flow.

In general, $C_{\mathrm{p}}$ coefficients at the rear wing upper surface are positive, they are negative at the lower rear wing surface, both contributing to the increased downforce. It is interesting to observe a strong and abrupt gradient in $C_{\mathrm{p}}$ coefficients at the lower rear wing surface, in agreement with McBeath (2011). As the wing mounting height increases, $C_{\mathrm{p}}$ coefficients at the lower surface of the wing become more negative, resulting in larger pressure differences thus enhancing the downforce. Simultaneously, the $C_{\mathrm{p}}$ coefficients at the upper surface become more positive creating the same effect. While these results indicate that the overall automobile traction and stability are anticipated to improve with increasing the rear wing height, this is further analyzed for the overall lift force coefficient of the automobile with the rear wing in place at different wing heights, Figure 16.

As reported in Figure 15 as well, the downforce (negative lift force) created by the rear wing increases with increasing the rear wing height. Simultaneously, the upforce (positive lift force) created by the automobile increases from $b / H=0.17$ to $b / H=0.39$, when it starts to slightly decrease. Those results combined yield an optimal rear wing height of $b / H=0.61$ with respect to the lift force. In order to determine an optimal rear wing height with

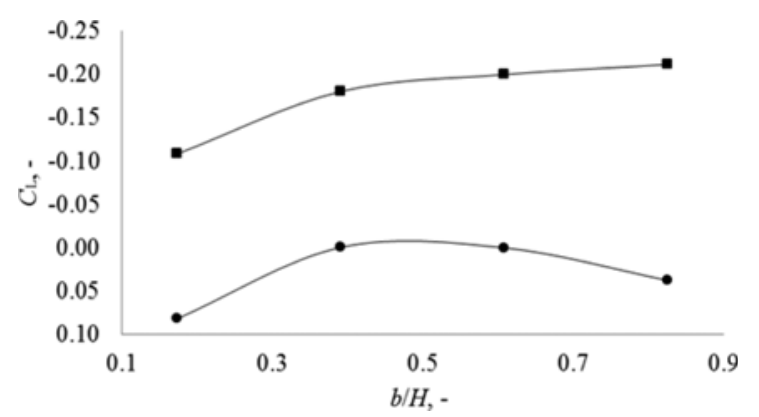

Figure 16. Contribution of the automobile body and the rear wing to the overall lift force coefficient; dots are CFD results for the automobile, squares are CFD results for the rear wing, solid lines are the best fit approximations. 


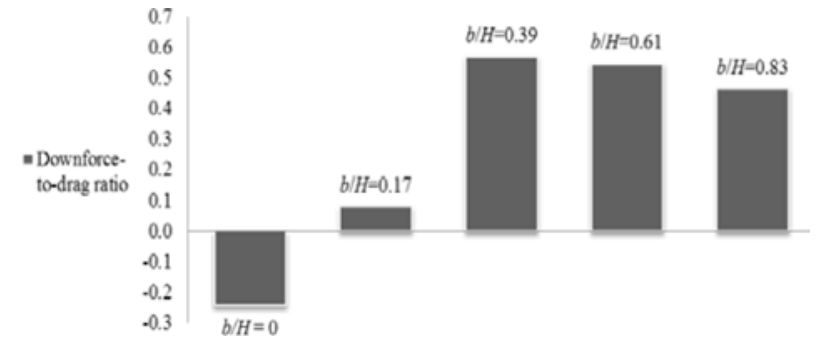

Figure 17. Downforce-to-drag ratio for every tested configuration.

respect to drag and lift force combined, the downforce-todrag ratio is determined, Figure 17. The optimal configuration is considered to be the one with the large downforce along with as small as possible drag force.

Without the rear wing, the downforce-to-drag ratio is negative and equal to -0.24 indicating the adverse upforce for this configuration. In all tests with the rear wing in place, there is a positive downforce-to-drag ratio indicating positive influence of the rear wing on the automobile traction and stability. An optimal rear wing position with respect to the downforce-to-drag ratio is for $b / H=0.39$ with the maximum observed downforce-to-drag ratio equal to 0.57 .

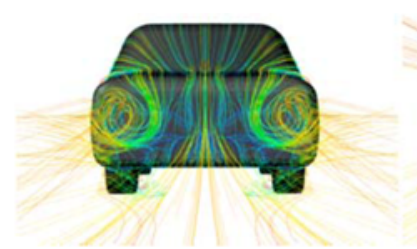

(a) $b / H=0$

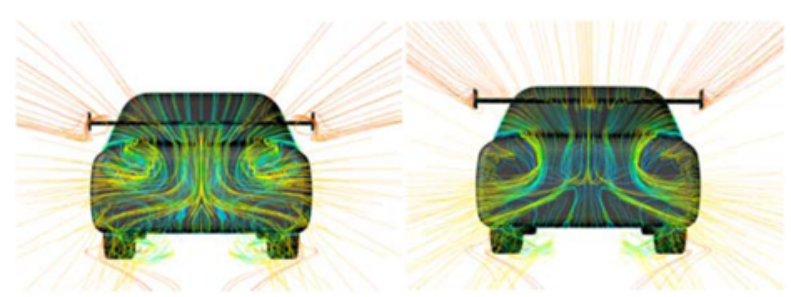

(c) $b / H=0.39$

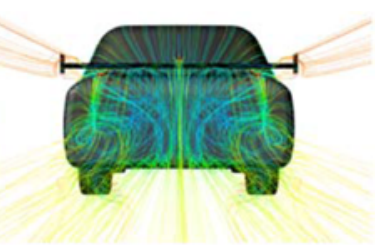

(b) $b / H=0.17$

(d) $b / H=0.61$

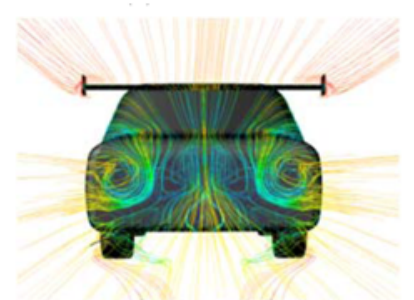

(e) $b / H=0.83$

Figure 18. Influence of the rear wing mounting height on vortex generation behind the automobile.
In addition, flow characteristics behind the automobile are analyzed for different rear wing heights as well that is based on the distribution of streamlines. Figure 18 shows the streamlines in the wake of the automobile when observed from the back of the vehicle (against the positive $x$-axis).

When there is no rear wing, two large vortices with horizontal axis of rotation (C-pillar vortices) develop at the trailing edge of the automobile roof (Bao, 2011). They are transferred with the main flow across the trunk and further downwind. The C-pillar vortices are not present for the rear wing placed in the lowest position $(b / H=0.17)$, while they are gradually restored for other (higher) positions of the rear wing. In particular, as the wing mounting height increases, the flow characteristics behind the car become more similar to the flow features in configuration without the rear wing. The $\mathrm{C}$-pillar vortices are restored to the largest extent for $b / H=0.83$.

\section{CONCLUSION}

Influence of the rear wing mounting height on the automobile aerodynamics is computationally studied by using the Reynolds-averaged-Navier-Stokes equations, standard $k-\varepsilon$ turbulence model and standard wall functions assuming the steady viscous fluid flow. An optimal wind incidence angle of the used rear wing is determined to be $18 \mathrm{deg}$ and it is constant with respect to the streamlines in all tested configurations. Without the rear wing in place, the automobile lift force is observed to be positive (upforce), while the downforce is obtained for all configurations with the rear wing in place thus indicating favorable effects on vehicle dynamics. The rear wing increases the automobile drag that is not favorable with respect to the automobile fuel consumption. Nevertheless, this drawback is not that important, as the rear wing considerably benefits the automobile traction and stability. An optimal rear wing position is determined to be at $39 \%$ of the height between the upper surface of the automobile trunk and the automobile roof. At this position, a maximum automobile downforce-to-drag ratio equal to 0.57 is obtained. Flow characteristics are studied behind the automobile by observing streamlines in the automobile wake. In this area, two large vortices with horizontal axis of rotation (C-pillar vortices) develop when there is no rear wing. Those vortices are not present when the rear wing is placed close to the upper trunk surface, while they gradually redevelop with the rear wing higher up from the trunk. Future work would need to address transient wind conditions, various wind incidence angles, different types of vehicles, all combining computational approach with laboratory and field experiments.

ACKNOWLEDGEMENT-The authors acknowledge support of the Croatian Science Foundation. 


\section{REFERENCES}

Bao, H. (2011). Study the effect of rear spoiler on car aerodynamic characteristics. The 6th Int. Conf. Computer Science and Education (ICCSE 2011), SuperStar Virgo, Singapore.

Carfolio (2015). http://www.carfolio.com/specifications

Chien-Hsiung, T., Lung-Ming, F., Chang-Hsien, T., YenLoung, H. and Jik-Chang, L. (2009). Computational aero-acoustic analysis of a passenger car with rear spoiler. Applied Mathematics Modelling 33, 9, 36613673.

Corin, R. J., He, L. and Dominy, R. G. (2008). A CFD investigation into the transient aerodynamic forces on overtaking road vehicle models. J. Wind Engineering and Industrial Aerodynamics 96, 8-9, 1390-1411.

Diasinos, S. and Gatto, A. (2008). Experimental investigation into wing span and angle-of-attack effects on sub-scale race car wing/wheel interaction aerodynamics. Exp. Fluids 45, 3, 537-546.

Elofsson, P. and Bannister, M. (2002). Drag reduction mechanisms due to moving ground and wheel rotation in passenger cars. SAE Paper No. 2002-01-0531.

Fackell, J. F. and Harvey, J. K. (1975). The aerodynamics of an isolated road wheel. AIAA Proc. the 2nd Symp. Aerodynamics of Sports and Competition Automobiles, Los Angeles, California, USA, 119-125.

Ferziger, J. H. and Perić, M. (2002). Computational Methods for Fluid Dynamics. 3rd edn. Springer-Verlag. Berlin, Germany.

Guilmineau, E. (2008). Computational study of flow around a simplified car body. J. Wind Engineering and Industrial Aerodynamics 96, 6-7, 1207-1217.

Ha, J., Jeong, S. and Obayashi, S. (2011). Drag reduction of a pickup truck by a rear downward flap. Int. J. Automotive Technology 12, 3, 369-374.

Hu, X., Zhang, R., Ye, Y., Yan, X. and Zhao, Z. (2011). Influence of different diffuser angle on sedan's aerodynamic characteristics. Physics Procedia, 22, 239245.

Kang, S. O., Jun, S. O., Park, H. I., Song, K. S., Kee, J. D., Kim, K. H. and Lee, D. H. (2012). Actively translating a rear diffuser device for the aerodynamic drag reduction of a passenger car. Int. J. Automotive Technology 13, 4, 583-592.

Katz, J. (1996). Race Car Aerodynamics. Bentley Publishers. Cambridge, UK.

Kozmar, H., Butler, K. and Kareem, A. (2012). Transient cross-wind aerodynamic loads on a generic vehicle due to bora gusts. J. Wind Engineering and Industrial Aerodynamics, 111, 73-84.

Kozmar, H., Butler, K. and Kareem, A. (2015). Downslope gusty wind loading of vehicles on bridges. J. Bridge Engineering 20, 11, 04015008-1-04015008-11.

Launder, B. E. and Spalding, D. B. (1974). The numerical computation of turbulent flows. Computer Methods in Applied Mechanics and Engineering 3, 2, 269-289.

McBeath, S. (2011). Competition Car Aerodynamics. Haynes Publishing, Bristol, UK.

Norwazan, A. R., Khalid, A. J., Zulkiffli, A. K., Nadia, O. and Fuad, M. N. (2012). Experimental and numerical analysis of lift and drag force of sedan car spoiler. Applied Mechanics and Materials, 165, 43-47.

Rizal, E. M., Firdaus, M., Ramlan, K., Shahriman, A., Faizal, M., Hanif, M. and Amir, G. (2012). Aerodynamics of ARTeC's PEC 2011 Emo-C car. Procedia Engineering, 41, 1775-1780.

Song, K. S., Kang, S. O., Jun, S. O., Park, H. I., Kee, J. D., Kim, K. H. and Lee, D. H. (2012). Aerodynamic design optimization of rear body shapes of a sedan for drag reduction. Int. J. Automotive Technology 13, 6, 905-914.

West, G. S. and Apelt, C. J. (1982). The effects of the tunnel blockage and aspect ratio on the mean flow past a circular cylinder with Reynolds numbers between $10^{4}$ and $10^{5}$. J. Fluid Mechanics, 114, 361-377. 